\title{
Audiological and Electrophysiological Evaluation of Children With Acquired Immunodeficiency Syndrome (AIDS)
}

\author{
Carla Gentile Matas ${ }^{1}$, Renata Aparecida Leite ${ }^{2}$, \\ Fernanda Cristina Leite Magliaro ${ }^{2}$ \\ and Isabela Crivellaro Gonçalves ${ }^{1}$
}

\begin{abstract}
${ }^{1}$ Department of Physiotherapy, Speech-Language and Hearing Science and Occupational Therapy; ${ }^{2}$ Department of Experimental Physiopathology; School of Medicine, University of São Paulo, São Paulo, SP, Brazil
\end{abstract}

\begin{abstract}
We examined the peripheral auditory system and the auditory brainstem pathway of children with Acquired Immunodeficiency Syndrome (AIDS). One hundred and one children, 51 with AIDS diagnosis and 50 normal children were evaluated. Audiological assessment included immittance measures, pure tone and speech audiometry and auditory brainstem response (ABR). The children with AIDS more frequently had abnormal results than did their matched controls, presenting either peripheral or auditory brainstem impairment. We suggest that AIDS be considered a risk factor for peripheral and/or auditory brainstem disorders. Further research should be carried out to investigate the auditory effects of HIV infection along the auditory pathway.
\end{abstract}

Key Words: Children; Hearing; Auditory Brainstem Response (ABR), HIV.

The acquired immunodeficiency syndrome (AIDS) is caused by the human immunodeficiency virus (HIV), an etiological agent responsible for severe impairments in the immune response of the affected individual. People with AIDS present a progressive immunological deficit, with increasing susceptibility to opportunistic infections.

In the vast majority of cases of HIV infected children, the virus is acquired during the perinatal period [1], when the infected mother transmits the virus to the fetus or newborn. The first suspected cases of children with an illness suggestive of AIDS were described in 1982, and in 1983 the first case reports of children infected during the perinatal period were published. Since then, much research has been done on the clinical evolution of HIV infection in this population, based on the hypothesis that this infection may be even more aggressive in children, with shorter latency periods between infection and the first symptoms, and a shorter survival period after the manifestation of symptoms, when compared to the adult population [2].

Children with AIDS are susceptible to a number of infections, including upper airway infections, especially sinusitis, external and middle ear otitis [3,4]. External and middle ear otitis may cause temporary peripheral hearing loss; it should be identified and properly treated as soon as possible.

The most common otologic manifestations of AIDS include external and middle ear otitis, sensorineural hearing loss, conductive hearing loss, mastoiditis, tympanic

Received on 5 January 2006; revised 18 July 2006.

Address for correspondence: Dr. Carla Gentile Matas. Avenida Divino Salvador 107-32, Planalto Paulista, Zip code: 04078-010 São Paulo, SP, Brazil. E-mail: cgmatas@usp.br. Phone: (+55 11) 50512217 / 91444240 .

The Brazilian Journal of Infectious Diseases 2006;10(4):264-268. (C) 2006 by The Brazilian Journal of Infectious Diseases and Contexto Publishing. All rights reserved. membrane perforation, colesteatoma and recurrent middle ear otitis with effusion. Hearing loss associated with AIDS, either sensorineural or central, may occur as a direct result of the effects of the virus on the central nervous system, on the peripheral auditory nerve, or as secondary effects to iatrogenic causes after ototoxic drug administration, infections (criptococcus meningitis, toxoplasmosis, viral or bacterial, cytomegalovirus, herpes) and central nervous system neoplasms [3].

Sensorineural hearing loss can affect $21 \%$ to $49 \%$ of HIV positive and AIDS subjects, typically causing worse losses for high frequency perception, indicating that there is a need for more studies about auditory pathway injuries caused by HIV [5]. Many studies have reported electrophysiological abnormalities in auditory brainstem response (ABR) and cognitive auditory potential (P300) at early stages of the disease, before the manifestation of clinical symptoms (such as neurological impairment and cognitive deficits), characterizing the demential complex of AIDS [6,7].

Published studies have emphasized the effects of HIV on the auditory evoked potentials of both children and adults. The generation of ABR waves depends on the temporal synchrony of neuronal activities in order to conduct auditory information. For this reason, damage to the central auditory system due to HIV directly affecting cortical and subcortical areas is possible, causing alterations in ABR waves [8].

Since the integrity of the peripheral and central auditory nervous system is essential for the acquisition and the development of language, speech and learning, it is mandatory that auditory abnormalities, both peripheral and central, be identified and treated as soon as possible, improving life quality and allowing effective communication by children with AIDS, even though the prognosis for this evolutive disease is poor in most cases. We made an audiologic evaluation of children with AIDS and controls, documenting both peripheral and auditory brainstem disorders. 


\section{Material and Methods}

The study was approved by the Ethics Committee for Research Projects of the Clinical Board of the Clinical Hospital of the School of Medicine of University of São Paulo, process number 070/00, and was carried out at the Speech-Language and Hearing Science Laboratory on Auditory Evoked Potentials of the Speech-Language and Hearing Science Course of the School of Medicine of the University of São Paulo.

The study comprised audiologic and electrophysiologic evaluations of 101 children, 51 with an AIDS diagnosis and 50 age-matched controls without AIDS. All children's parents or legal guardians signed the informed consent term approved by the Ethics Committee of the School of Medicine of the University of São Paulo.

The control group children were referred from an elementary school. The selection of this group was based on the clinical history of the subjects, provided by their parents or caregivers. Children with family history and/or risk factors for HIV infection (perinatal transmission, blood transfusion, injectable drug use, sexual intercourse and occupational accidents) [9] were excluded.

The study group children were referred from various institutions, including children from AIDS-support clinics. The clinical history was supplied by the parents or caregivers in order to provide personal and parental data concerning HIV infection, pre, peri and post-natal risk factors for hearing loss, occurrence of otitis and ear suppuration, and complaints concerning hearing difficulties and/or delay in the acquisition and development of language and speech. The time of exposure to the virus, transmission and clinical manifestations of AIDS were not considered for the selection of the study group, since it was very difficult to assure the presence of children with AIDS at the clinic for evaluation procedures.

Fifty-one children with AIDS were divided into two groups, according to their ages and to the audiologic procedures used. Age was considered, based on literature reports that young children are more susceptible to infections due to the immaturity of their immunological systems [2].

Group I included 26 children with AIDS ranging from 3 to 6 years of age (mean age 4 years, 8 months), with 10 males and 16 females. Twenty-five children without AIDS ranging in age from 3 to 6 years (mean age 5 years, 1 month), with 14 males and 11 females, were included in the group I control.

Group II comprised 25 children with AIDS, ranging in age from 7 to 10 years (mean age 8 years, 5 months), with 12 males and 13 females. Twenty-five children without AIDS, ranging from 7 to 10 years of age (mean age 8 years, 4 months), 15 males and 10 females, were included in the group II control. After obtaining clinical history information, the external ear canal was inspected with a Heine otoscope, followed by an audiologic evaluation in a sound-proof booth. The audiologic assessment used different procedures according to the age of the child and his/her clinical status. The procedures were the following:

1) Pure tone threshold audiometry (Play Conditioned for group I, Traditional for group II) including octave frequencies between 0.5 and $4 \mathrm{kHz}$ for group I, and octave frequencies between 0.25 and $8 \mathrm{kHz}$ for group II, using a Grason-Stadler GSI 10 and GSI 16 audiometers.

2) Acoustic immittance measures, using a $226 \mathrm{~Hz}$ probe tone for tympanometry and acoustic reflexes, with a Grason-Stadler GSI 33 equipment.

3) Auditory brainstem response (ABR), using 0.1 millisecond rarefaction click stimuli presented through earphone receivers for each ear at $80 \mathrm{~dB} \mathrm{HL}$, at a presentation rate of 19.0 stimuli/ second, totalizing 2000 stimuli, with Traveler Express - BIOLOGIC portable equipment.

Given the age of the participants of this study (3 to 10-yearold children), the only electrophysiological test that was carried out was the ABR. The maturation of the brainstem is complete at around eight months of age, while the other potential evoked auditory measures (middle latency response and late latency response or congnitive potential - P300) assess auditory subcortical and cortical areas that are only mature at about 8-10 [10] and 14 years of age [11] respectively, which made the clinical application of this evaluation impracticable.

The audiologic assessment was considered normal when hearing thresholds were lower than or equal to $15 \mathrm{~dB}$ NA (conventional and conditioned pure tone audiometry), the tympanogram was type A and there were acoustic reflexes (acoustic immitance measures). Normative latency data was referred to during ABR interpretations [12].

\begin{tabular}{|lcccc|}
\hline $\begin{array}{l}\text { Confidence } \\
\text { intervals }\end{array}$ & Limits & Wave I & WaveIII & Wave V \\
CI 95\% & Inferior & 1.4478 & 3.5278 & 5.4500 \\
& Superior & 1.4954 & 3.5978 & 5.5180 \\
Confidence & Limits & I*(I-III) & I(III-V) & I(I-V) \\
intervals & & & & \\
CI 95\% & Inferior & 2.0888 & 1.9193 & 4.0098 \\
& Superior & 2.0936 & 1.9231 & 4.0150 \\
\hline
\end{tabular}

* I= interpeak.

Audiological and electrophysiological results, in both groups, that did not meet the specified normality criteria described above were considered deviant and were later classified as peripheral disorders, auditory brainstem disorders or mixed disorders.

Subjects were considered as having peripheral auditory disorders if they showed conductive or sensorineural hearing losses detected by conventional or conditioned pure tone audiometry and acoustic immitance measures, and if they had, in the case of conductive losses, a delay of the absolute latency of waves I, III and V and normal interpeaks I-III, I-V and III-V, 
and, in the case of sensorineural losses, normal absolute latencies of waves I, III and V and interpeaks I-III, I-V and III-V on ABR measures [13]. Brainstem auditory disorders were considered when auditory thresholds obtained by conventional or conditioned pure tone audiometry were normal and the ABR results showed either a delay in the absolute latencies of waves III and V, with increase of I-III and I-V interpeaks (suggesting low brainstem auditory disorder), or a delay in the absolute latency of wave $\mathrm{V}$, with increase of III-V and I-V interpeaks (suggesting high brainstem auditory disorders) [13]. Finally, mixed disorders were classified by the combined presence of peripheral and brainstem auditory disorders, showing conductive hearing loss with delay of the absolute latency of waves I, III and V and increased I-III, I-V and/or III$\mathrm{V}$ interpeaks, and sensorineural hearing loss with delay of the absolute latency of waves III and/or V, and increased I-III, I-V and/or III-V interpeaks. Specialized audiologists carried out the clinical history and the audiologic and electrophysiologic evaluations from 2001 and 2003.

\section{Statistical analysis}

The sample size was determined considering the central limit theorem and great numbers laws, which showed that 25 subjects per group would allow the data distribution to approximate a normal distribution. Statistical analysis was carried out using a two proportion equality test in order to compare a specific variable in two different samples; the following tests were designed:

\section{Comparison}

Group I Control Group I Study

Group II Control Group II Study

Group I Study Group II Study

The comparison of the results obtained from the study and control groups did not match the subjects individually by age, but instead, matched them as groups.

\section{Results}

Auditory disorders in children with AIDS, from 3 to 6 years of age

First, each subject's results were classified as normal or abnormal. They were considered normal when the results were within normal limits in all tests (audiologic and electrophysiologic), and abnormal when the results were not within normal limits in one or more of the procedures (audiological and/or electrophysiological).

Young children with AIDS had a greater frequency of auditory problems (Table 1). All the young control children had auditory findings within normal limits (100\%), while only $58 \%$ of the AIDS children had normal results. The difference between the groups was significant $(\mathrm{P}<0.001)$.

Abnormal results were classified according to the type of the disorder found: peripheral auditory disorders concerning inner or middle ear conditions (resulting from middle ear otitis), auditory brainstem disorders (low and/or high), and mixed disorders involving the presence of peripheral (impairment concerning middle ear or inner ear conditions) and auditory brainstem disorders.

Table 1. Comparison of normal/abnormal auditory test results for children with and withoutAIDS, ranging from 3 to 6 years old

\begin{tabular}{lcccc}
\hline & $\begin{array}{c}\text { Control group } \\
(\mathbf{N}=\mathbf{2 5})\end{array}$ & \multicolumn{2}{c}{$\begin{array}{c}\text { AIDS group } \\
\mathbf{( N = 2 6 )}\end{array}$} \\
\cline { 2 - 5 } & $\mathbf{N}$ & $\mathbf{\%}$ & $\mathbf{N}$ & $\mathbf{\%}$ \\
\hline Normal & 25 & $100 \%$ & 15 & $58 \%$ \\
Abnormal & 0 & $0 \%$ & 11 & $42 \%$ \\
\hline
\end{tabular}

$\mathrm{P}<0.001^{*}$

The diagnosis of middle ear impairment was based on audiological and immittance measures, and delayed absolute latencies with normal interpeaks in ABR. All these children were referred to otolaryngologists (Table 2). Among the auditory disorders found in these children, $45 \%$ were peripheral, $10 \%$ were auditory brainstem disorders and the other $45 \%$ were classified as mixed disorders.

Table 2. Frequency of auditory disorders for children with AIDS ranging from 3 to 6 years old

\begin{tabular}{lcc}
\hline Auditory disorders & N & \% \\
\hline Peripheral auditory disorders & 5 & $45 \%$ \\
Auditory brainstem disorders & 1 & $10 \%$ \\
Mixed disorders & 5 & $45 \%$ \\
\hline $\mathrm{P}=0.056$ (peripheral auditory disorders X & \\
brainstem disorders).
\end{tabular}

Auditory disorders in children with AIDS ranging from 7 to 10 years of age

All control children 7 to 10 years old had auditory test results within normal limits (100\%), while only 68\% of the AIDS-affected children in this age group had normal results. The difference between the groups was significant (Table 3, $\mathrm{P}=0.002$ ).

Table 3. Comparison of normal/abnormal auditory findings for children with AIDS, ranging from 7 to 10 years old, and controls

\begin{tabular}{lcccc}
\hline & $\begin{array}{c}\text { Control group } \\
(\mathbf{N}=\mathbf{2 5})\end{array}$ & \multicolumn{2}{c}{$\begin{array}{c}\text { AIDS group } \\
(\mathbf{N}=\mathbf{2 5})\end{array}$} \\
\cline { 2 - 5 } & $\boldsymbol{N}$ & $\%$ & $\boldsymbol{N}$ & $\%$ \\
\hline Normal & 25 & $100 \%$ & 17 & $68 \%$ \\
Abnormal & 0 & $0 \%$ & 8 & $32 \%$ \\
\hline $\mathrm{P}<0.002^{*}$. & & & & \\
\hline
\end{tabular}

Six of the eight auditory disorders in the 7-10 year old AIDS-affected children were peripheral and the other two were auditory brainstem disorders. None of the children 
had mixed disorders. Significant differences among peripheral, auditory brainstem and mixed disorders were found (Table 4).

Table 4. Frequencies of auditory disorders in children with AIDS ranging from 7 to 10 years old

\begin{tabular}{lll}
\hline Auditory disorders & N & \% \\
\hline Peripheral auditory disorders & 6 & $75 \%$ \\
Auditory brainstem disorders & 2 & $25 \%$ \\
Mixed disorders & 0 & $0 \%$ \\
\hline
\end{tabular}

$\mathrm{P}=0.046$ (peripheral auditory disorders $\mathrm{x}$ auditory brainstem disorders). $\mathrm{P}=0.002$ (peripheral auditory disorders $\mathrm{x}$ mixed disorders). $\mathrm{P}=0.131$ (auditory brainstem disorders $\mathrm{x}$ mixed disorders).

Comparison of auditory disorders in children with AIDS ranging from 3 to 6 and from 7 to 10 years of age

The frequency of peripheral auditory disorders, auditory brainstem disorders and mixed disorders was compared between the age groups (Table 5). Mixed disorders were more frequent in the younger age group $(\mathrm{P}=0.026)$.

\section{Discussion}

Most papers in the specialized literature address auditory disorders for a wide range of ages and sometimes present the results of audiologic evaluation in adult and pediatric populations together. Though it was difficult to find similar studies for comparison, we maintained age range grouping because of reports that younger children are more susceptible to infections, since their immunological system is not yet mature [6].

Occurrence of auditory disorders in children ranging from 3 to 6 years of age, who have AIDS

The proportion of normal audiologic and electrophysiologic test results was significantly higher in the three - six-year-old controls than in those with AIDS (Table 1). Other studies have also demonstrated a higher incidence of auditory disorders in children with AIDS [14-16].

Peripheral auditory disorders and mixed disorders (both peripheral or auditory brainstem disorders) were the most prevalent results among children with AIDS ranging from three to six years old. However, the differences were not significant (Table 2).

According to the specialized literature, both peripheral and auditory brainstem impairments can occur in children with AIDS. Most of the studies report one of these types of impairment, some describing peripheral auditory disorders resulting from middle ear impairment and others describing auditory brainstem disorders. Some authors have reported a higher incidence of middle ear dysfunction in children with AIDS [14]. We observed peripheral and/or brainstem auditory impairments in children with AIDS, in agreement with other researchers $[17,18]$. The peripheral disorders in these subjects were due middle ear dysfunction. Inner ear dysfunction was not observed.

Brainstem auditory disorders have been documented by various authors, who emphasized that infection of the central nervous system by HIV in the pediatric population causes central auditory dysfunctions $[4,19]$. Children with AIDS seem to be more susceptible to central nervous system involvement, which leads to early central auditory processing disorders [14].

Occurrence of auditory disorders in children with AIDS ranging from 7 to 10 years of age

Results within normal limits were much more frequent in the control group than in the 7-10-year-old children with AIDS, and this difference was significant. Abnormal results were also more frequently observed in the group of children with AIDS, when compared to the control group of the same age (Table 3). Peripheral auditory disorders (resulting from middle or inner ear dysfunctions) were the most frequent abnormality observed in this age group (Table 4). Only one child of this group had an auditory disorder resulting from inner ear impairment (unilateral sensorineural hearing loss at $8 \mathrm{kHz}$ ), while the others presented middle ear dysfunction as a consequence of otitis media (Table 4). The specialized literature also highlights the predominance of middle ear dysfunction in these subjects [14].

The most common reason for sensorineural hearing loss in children with AIDS is the use of ototoxic drugs for the treatment of HIV infection [4]. Studies have described a high frequency sensorineural hearing loss after the administration of anti-retroviral drugs in these patients [20,21].

Comparative study of auditory disorders found in children with AIDS ranging from 3 to 6 and from 7 to 10 years old

This comparison allowed us to note that the type of disorder observed in children with AIDS (peripheral and auditory brainstem disorders) was influenced by age, revealing significant differences between the two age groups; a greater number of either peripheral or auditory brainstem disorders was observed in children from 3 to 6 years of age than in children from 7 to 10 years of age (Table 5). A previous study had also emphasized the greater susceptibility of central nervous system involvement in children with AIDS, leading to early central

Table 5. Comparison of different types of auditory disorders in children with AIDS, distributed according to age

\begin{tabular}{|c|c|c|c|c|c|c|c|}
\hline \multirow[t]{2}{*}{ AIDS } & \multicolumn{2}{|c|}{ Peripheral auditory disorders } & \multicolumn{2}{|c|}{ Auditory brainstem disorders } & \multicolumn{2}{|c|}{ Mixed disorders } & \multirow[t]{2}{*}{ Total } \\
\hline & $\mathbf{N}$ & $\%$ & $\mathbf{N}$ & $\%$ & $\mathbf{N}$ & $\%$ & \\
\hline 3 to 6 years & 5 & $45 \%$ & 1 & $10 \%$ & 5 & $45 \%$ & 11 \\
\hline 7 to 10 years & 6 & $75 \%$ & 2 & $25 \%$ & 0 & $0 \%$ & 8 \\
\hline $\mathrm{P}$ & 0.198 & & 0.348 & & 0.026 & & \\
\hline
\end{tabular}


auditory deficits in this pediatric population, including auditory brainstem disorders [14]. No studies aimed at comparing the types of auditory disorders in children with AIDS according to their age were found in the specialized literature.

Progressive abnormalities have been found in the auditory brainstem response of patients with AIDS, following disease progression. This disease may produce subclinical pathologic changes in the cochlear nerve and brainstem [22].

We conclude that children with AIDS can have either peripheral (mainly conductive hearing loss resulting from middle ear involvement, as a consequence of otitis media) or brainstem auditory disorders. Consequently, investigation of the auditory pathway as a whole should be mandatory for such children. Further research is needed in order to better understand the effect of HIV infection on peripheral and central auditory systems (brainstem pathway) in the pediatric population with AIDS. The fact that different types of auditory disorders were observed in different age ranges points to the need for a follow-up program for children with AIDS, in order to their test them periodically.

Critical analysis of the results allowed the following conclusions:

1) Children with AIDS from 3 to 6 years of age showed:

- a higher proportion of abnormal results than the control group;

- isolated peripheral auditory disorders or peripheral auditory disorders coexisting with auditory brainstem impairments were more frequent than isolated auditory brainstem disorders;

- more peripheral and auditory brainstem disorders occurred simultaneously than in children with AIDS from 7 to 10 years of age.

2) Children with AIDS from 7 to 10 years of age showed:

- a higher proportion of abnormal results than the control group;

- isolated peripheral auditory disorders more frequent than auditory brainstem disorders and peripheral disorders associated with auditory brainstem disorders;

- more peripheral and auditory brainstem disorders occurring in isolation than in children with AIDS from 3 to 6 years of age.

\section{References}

1. Orloff S.L., Simonds R.J., Steketee R.W., St. Louis M.E. Determinants of perinatal HIV-1 transmission. Clin Obstet Gynecol 1996; 39(2):386-95.

2. Sculerati N., Borkowsky W. Pediatric human immunodeficiency virus infection: an otolaryngologist's perspective. J Otolaryngol 1990;19(3):182-8.

3. Gold S., Tami T.A. Otolaryngological manifestations of HIV/ AIDS. Seminars in Hearing 1998;19(2): 165.

4. Matkin N.D., Diefendorf A.O., Erenberg A. Children: HIV/AIDS and hearing loss. Seminars in Hearing 1998;19(2): 143-3.

5. Roland J.T.Jr., Alexiades G., Jackman A.H., et al. Cochlear implantation in human immunodeficiency virus - infected patients. Otology \& Neurotology 2003;24(6):892-5.

6. Smith T., Jakobsen J., Gaub J., et al. Clinical and electrophysiological studies of human immunodeficiency virus - soropositive men without AIDS. Ann. Neurol 1988;23:295-7.

7. Ollo C., Johnson R., Grafman J. Signs of cognitive change in HIV disease: an event-related brain potential study. Neurology 1991;41:209-15.

8. Bankaitis A.E., Christensen L.A., Murphy G., Morehouse C. R. HIV/AIDS and auditory evoked potentials. Seminars in Hearing 1998;19(2):175,177-93.

9. Friedland G.H., Klein R.S. Transmission of the human immunodeficiency virus. $\mathrm{N}$ Engl J Med 1987;317(18):1125-35.

10. Hall III J.W. Handbook of Auditory Evoked Responses. Allyn \& Bacon, Boston, 1992.

11. Buschwald J.S. Comparison of plasticity in sensory and cognitive processing systems. Clinical Perinatology 1990;17(1):57-66.

12. Flabiano F.C., Leite R.A., Matas C.G. Audiometria de tronco encefálico em adultos audiologicamente normais: comparação das latências absolutas das ondas I, III, V, interpicos I-III, IIIV, I-V, amplitude das ondas I, III, V e relação da amplitude V/ I, obtidas em dois equipamentos diferentes. Acta AWHO 2002;21(1):[13 figures].

13. Matas C.G. Interpretando uma Audiometria de Tronco Encefálico. In: Gama M.R. org. Resolvendo casos em Audiologia. São Paulo: Plexus, 2001.

14. Madriz J.J., Herrera G. Human immunodeficiency virus and acquired immune deficiency syndrome AIDS - related hearing disorders. J Am Acad Audiol 1995;6(5): 358-64.

15. Layton T.L., Davis-McFarland E. Pediatric human immunodeficiency virus and acquired immunodeficiency syndrome: an overview. Semin Speech Lang 2000;21(1):7-17.

16. Soucek S., Michaels L. The ear in the acquired immunodeficiency syndrome: II.Clinical and audiologic investigation. Am J Otol 1996;17(1):35-9.

17. Belman A. L., Ultmann M. H., Horoupian D. et al. Neurological complications in infants and children with acquired immune deficiency syndrome. Ann Neurol 1985;18:560-6.

18. Birchall M. A., Wight R. G., French P. D., et al. Auditory function in patients infected with the human immunodeficiency virus. Clin Otolaryngol Allied Sci 1992;17(2):117-21.

19. Schmitt B., Seeger J., Jacobi G. EEG and evoked potentials in HIV infected children. Clin Electroencephalogr 1992;23(3):111-7.

20. Christensen L.A., Morehouse C.R., Powell T.W., et al. Antiviral therapy in a child with pediatric human immunodeficiency virus (HIV): case study of audiologic findings. J Am Acad Audiol 1998;9(4):292-8.

21. Mata Castro N., Yebra Bango M., Tutor De Ureta P., et al. Hipoacusia e infección por el vírus de la inmunodeficiencia humana. Estudio de 30 pacientes. Rev Clin Esp 2000;200(5):271-4.

22. Reyes-Contreras L., Silva-Rojas A., Ysunza-Rivera A., et al. Brainstem auditory evoked response in HIV-infected patients with and without AIDS. Arch Med Res 2002;33(1):25-8. 